\title{
Lesão Visceral em Trauma Abdominal: Um Estudo Retrospetivo
}

\author{
Visceral Injury in Abdominal Trauma: A Retrospective Study
}

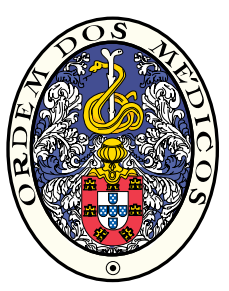

\author{
Sara LEITE ${ }^{1}$, António TAVEIRA-GOMES², Hugo SOUSA ${ }^{2}$ \\ Acta Med Port 2013 Nov-Dec;26(6):725-730
}

RESUMO

Introdução: O trauma abdominal é uma importante causa de morbi-mortalidade em todo o mundo, o que leva a que seja fulcral uma abordagem focada no rápido diagnóstico e tratamento. Este estudo tem como objetivos principais, por um lado, identificar dados epidemiológicos globais do trauma abdominal no Centro Hospitalar de São João e, por outro, estudar as lesões traumáticas, tratamento e outcome.

Material e Métodos: Análise retrospetiva do processo clínico de todos os doentes admitidos com traumatismo abdominal, durante um período de cinco anos, num hospital central universitário.

Resultados: A média total de idades foi de 42,6 anos e o sexo masculino, foi o mais afetado (74,2\%). A maior parte dos doentes tinha um Revised Trauma Score > 4, na admissão. Os mecanismos traumáticos mais frequentes foram fechados por acidente de viação (39,9\% em ocupantes de veículo e $10,7 \%$ por atropelamento) e queda (25,5\%). O trauma perfurante, por lesão de arma branca ou de fogo, ocorreu em apenas $12,3 \%$ dos casos. As lesões de víscera oca ocorreram mais neste contexto. Em 19,5\% dos casos ocorreu lesão de mais do que uma víscera abdominal. O tratamento conservador foi feito em $65,3 \%$ dos casos. A mortalidade total foi de $12 \%$, sendo nula nos casos de lesão penetrante.

Conclusão: O trauma abdominal resulta de acidentes de viação e quedas, mais frequentemente, sendo fechado na maioria dos casos. Os órgãos mais vezes afetados são maciços e o tratamento conservador. As lesões de víscera oca continuam a ser de difícil diagnóstico.

Palavras-chave: Estudos Retrospectivos; Ferimentos e Lesões; Portugal; Traumatismos Abdominais; Traumatismos Múltiplos.

\section{ABSTRACT}

Background: Abdominal trauma is a major cause of morbi-mortality all over the world which makes it essential an approach focused on rapid diagnosis and treatment. The main goals of this study are to identify global epidemiologic data of abdominal trauma in our tertiary trauma center and to study traumatic lesions, treatment and outcome.

Material and Methods: Retrospective analysis of the clinical file of all patients admitted with abdominal trauma, over a period of 5 years, in a tertiary trauma center.

Results: the total mean of ages was 42.6 years and the male gender was the most affected $(74.2 \%)$. At admission, most patients had a Revised Trauma Score $>4$. The mainly causes of trauma were blunt from motor-vehicle collisions ( $39.9 \%$ as motor-vehicle occupant and $10.7 \%$ from pedestrian collisions) and falls (25.5\%). Penetrating trauma, by abdominal stab wounds and gunshot wounds, occurred only in $12.3 \%$ of the cases. Hollow visceral injuries were more frequent in that context. In $19.5 \%$ of the cases multiple abdominal organ injury occurred. Conservative treatment was performed in $65.3 \%$ of the cases. Global mortality was $12 \%$, being null after penetrating lesions.

Conclusions: Abdominal trauma, more frequently, is the result of motor-vehicle crashes and falls, being blunt in the majority of the cases. The most affected organs are solid and the approach is conservative. Hollow visceral lesions continue to be of difficult diagnose. Keywords: Abdominal Injuries; Multiple Trauma; Portugal; Retrospective Studies; Wounds and Injuries.

\section{INTRODUÇÃO}

O trauma abdominal é uma importante causa de morbi-mortalidade em todo o mundo, ${ }^{1-4}$ o que leva a que seja fulcral uma abordagem focada no rápido diagnóstico e tratamento. ${ }^{5}$ Existem dois grandes grupos de mecanismos traumáticos: o fechado, mais prevalente, que inclui os acidentes de viação e quedas, entre outros, e o penetrante que abrange fundamentalmente as lesões por arma branca ou de fogo, ${ }^{6,7}$ cuja incidência tem aumentado na última década. ${ }^{6}$ As lesões intra-abdominais traumáticas podem verificar-se em vísceras sólidas, lesões mais frequentes e muitas vezes autolimitadas, ${ }^{6}$ ou em vísceras ocas. Estas apesar de menos prevalentes (cerca de $1 \%$ dos casos de trauma fechado), ${ }^{8}$ acarretam maior dificuldade diagnóstica, com atraso no tratamento e consequente agravamento da morbilidade e mortalidade.$^{9-11} \mathrm{O}$ fígado e o baço são os órgãos mais vezes lesados ${ }^{7,12} \mathrm{e}$, em até $10 \%$ dos casos, há lesões de víscera oca associadas. ${ }^{8}$ Os exames auxiliares mais utilizados em trauma são a Ecografia-FAST (Focused Assessment with Sonography in Trauma), portátil, rápida e não invasiva, ${ }^{13,14}$ que nos permite obter informação sobre a presença de líquido intraperitoneal, pleural ou pericárdi$\mathrm{CO}^{15} \mathrm{e}$ a tomografia computorizada (TC), o gold-standard no trauma fechado, ${ }^{5}$ que tem assumido um papel importante na decisão do tratamento não operatório de lesões hepáticas, esplénicas e renais. ${ }^{6,11}$ A Laparoscopia é um método rápido e minimamente invasivo, que permite o diagnóstico e

1. Departamento de Cirurgia. Faculdade de Medicina. Universidade do Porto. Porto. Portugal.

2. Serviço de Cirurgia Geral. Centro Hospitalar de São João/Faculdade de Medicina da Universidade do Porto. Porto. Portugal.

Recebido: 16 de Março de 2013 - Aceite: 09 de Setembro de 2013 | Copyright @ Ordem dos Médicos 2013 
tratamento de lesões. ${ }^{16}$ É mais comummente utilizada após trauma penetrante, para investigação de perfuração peritoneal em doentes hemodinamicamente estáveis. ${ }^{6,16} \mathrm{O}$ seu papel no trauma fechado ainda não se encontra bem esclarecido podendo ser uma hipótese a considerar em doentes estáveis, com suspeita de lesão intra-abdominal. ${ }^{17}$ Os estudos mais recentes afirmam que, em até $45 \%$ dos casos, o diagnóstico não é feito corretamente. ${ }^{17} \mathrm{~A}$ Laparotomia exploradora é indicada em doentes hemodinamicamente instáveis, com sinais de peritonite e perfuração de víscera $\mathrm{oca}^{3}$ e deixou de ser mandatória em contexto de trauma penetrante, pela alta taxa de laparotomias não terapêuticas (cerca de $38 \%) .{ }^{18} \mathrm{O}$ tratamento das lesões pode ser, por isso, não operatório, principalmente no caso de lesões hepáticas (em $70 \%$ dos casos sem aumento do risco de mortalidade) ${ }^{11}$ e esplénicas (fundamentalmente em doentes hemodinamicamente estáveis após trauma fechado), ${ }^{19}$ ou cirúrgico que, em $20 \%$ dos casos, ${ }^{6}$ tem como objetivos restabelecer a fisiologia e a hemostase, através da realização de ressecções essenciais e estomas temporários (cirurgia de controlo de danos). ${ }^{6}$

O presente estudo tem como objetivos principais, por um lado, identificar dados epidemiológicos globais do Trauma abdominal no Centro Hospitalar de S. João e, por outro, estudar as lesões traumáticas, tratamento e outcome.

\section{MATERIAL E MÉTODOS}

O estudo foi feito com base na análise retrospetiva do processo clínico de todos os doentes admitidos com traumatismo abdominal, durante um período de cinco anos (Maio de 2006 a Maio de 2011), num hospital central universitário. Foram recolhidos dados demográficos dos doentes, o revised trauma score à entrada, os métodos de abordagem diagnóstica e terapêutica, tempo de internamento e o destino de alta.

Foram consultados os relatórios de autópsia dos doentes falecidos, na Delegação do Norte do Instituto Nacional de Medicina Legal.

Foi feita análise dos dados em SPSS versão 19.0.

O estudo foi submetido e aprovado pela Comissão de Ética do Hospital de São João.

\section{RESULTADOS}

Entre Maio de 2006 e Maio de 2011, foram admitidos 326 doentes com trauma abdominal. A média de idades foi de 42,6 anos e o sexo masculino foi o mais afetado $(74,2 \%)$. Os mecanismos traumáticos mais frequentes foram o acidente de viação (39,9\% em ocupantes de veículo e 10,7\% por atropelamento) e a queda (25,5\%). A maior parte dos doentes tinha um revised trauma score $>4$ na admissão. As principais características demográficas, o score de

Tabela 1 - Caraterísticas demográficas, score de gravidade e outcome

\begin{tabular}{|c|c|c|c|c|}
\hline & $\begin{array}{c}\text { Trauma fechado } \\
n(\%)\end{array}$ & $\begin{array}{c}\text { Trauma Penetrante } \\
n(\%)\end{array}$ & $\begin{array}{c}\text { Não especificado } \\
n(\%)\end{array}$ & $\begin{array}{l}\text { Total } \\
n(\%)\end{array}$ \\
\hline Idade & 43,7 & & & \\
\hline
\end{tabular}

Género

$\begin{array}{rrrrr}\text { Masculino } & 203(62,3) & 32(9,8) & 7(2,1) & \mathbf{2 4 2}(\mathbf{7 4 4 , 2 )} \\ \text { Feminino } & 75(23) & 8(2,5) & 1(0,3) & \mathbf{8 4}(\mathbf{2 5}, \mathbf{8})\end{array}$

Mecanismo traumático*

Acidente viação

$165(50,6)$

Queda

$83(25,5)$

Arma branca

Arma fogo

Outros

$30(9,2)$
$245(75,2)$

$27(8,3)$
$35(10,7)$

$4(1,2)$

$1(0,3)$

$5(1,5)$
$165(50,6)$

$83(25,5)$

$22(6,7)$

$18(5,5)$

$38(11,7)$

Não especificado

$27(8,3)$

$241(73,9)$

$40(12,3)$

$6(1,8)$

287 (88)

Falecido

$37(11,3)$

0

$2(0,6)$

$39(11,9)$ 
gravidade à admissão e outcome encontram-se resumidos na Tabela 1.

O trauma fechado, fundamentalmente por acidente de viação e queda, foi o mais frequente ( $85,3 \%$ dos casos). As lesões de víscera sólida, nomeadamente do baço, fígado e rim (Tabela 2$)$, foram mais prevalentes $(91,9 \%$ do total de casos).

O trauma perfurante, por lesão de arma branca ou de fogo, ocorreu em apenas $12,3 \%$ dos casos. Apesar de raras, as lesões de víscera oca, quando comparadas com as lesões de víscera sólida, ocorreram mais neste contexto, com $66,7 \%$ das lesões do estômago e $25 \%$ das lesões do cólon por lesão de arma de fogo e com $26,9 \%$ das lesões do delgado por lesão de arma branca (Tabela 3).

Tabela 2 - Mecanismo traumático versus Víscera atingida
Em 19,5\% dos casos ocorreu lesão de mais do que uma víscera abdominal. $\mathrm{O}$ acidente de viação (como ocupante de veículo) foi a causa em $8,9 \%$ do número total das lesões associadas. As associações de lesão mais frequentemente encontradas foram entre o fígado e baço (5\%), o fígado e rim $(4,6 \%)$, baço e rim $(3,7 \%)$ e entre o delgado e cólon $(1,5 \%)$.

No que diz respeito aos exames auxiliares de diagnóstico, em $4,3 \%$ dos casos não foi especificado o exame utilizado. O exame imagiológico privilegiado foi a TC abdominal $(71,5 \%)$, com $96,6 \%$ dos doentes com um revised trauma score $>4$, seguida da ecografia abdominal (62,6\%). A lavagem peritoneal diagnóstica foi uma abordagem pouco utilizada $(0,6 \%$ dos casos) e em $4 \%$ dos casos procedeu-se

\begin{tabular}{llllll}
$\substack{\text { Mecanismo } \\
\text { Traumático }}$ & Baço* & Fígado & Rim & Estômago* & Delgado* Cólon* \\
\hline
\end{tabular}

\section{Queda}

$\%$ dentro mecanismo traumático $\%$ dentro víscera $\%$ do total 24
$28,9 \%$
$22,2 \%$ $22,2 \%$
$7,4 \%$

\section{Acidente de viação}

Ocupante de veículo

$\%$ dentro mecanismo traumático $\%$ dentro víscera $\%$ do total

\section{Atropelamento}

54
$41,5 \%$
$50 \%$
$16,6 \%$

$\begin{array}{rr}51 & 27 \\ 39,2 \% & 20,8 \% \\ 47,2 \% & 32,5 \% \\ 15,6 \% & 8,3 \%\end{array}$

1
$0,8 \%$
$16,7 \%$
$0,3 \%$

2
$2,4 \%$
$7,7 \%$
$0,6 \%$

$n$

$\%$ dentro mecanismo traumático \%dentro víscera $\%$ do total

\section{Lesão arma branca}

$\begin{array}{rrr}11 & 10 & 12 \\ 31,4 \% & 28,6 \% & 34,3 \% \\ 10,2 \% & 9,3 \% & 14,5 \% \\ 3,4 \% & 3,1 \% & 3,7 \%\end{array}$

0
$0 \%$
$0 \%$
$0 \%$

$\begin{array}{rr}2 & 3 \\ 5,7 \% & 8,6 \% \\ 7,7 \% & 18,8 \% \\ 0,6 \% & 0,9 \%\end{array}$

$\%$ dentro mecanismo traumático $\%$ dentro víscera $\%$ do total

\section{6}

$$
1,8 \%
$$

Lesão arma fogo

$$
\begin{array}{r}
n \\
\% \text { dentro mecanismo traumático } \\
\% \text { dentro víscera } \\
\% \text { do total }
\end{array}
$$

$\begin{array}{rrr}5 & 8 & 3 \\ 27,8 \% & 44,4 \% & 16,7 \% \\ 4,6 \% & 7,4 \% & 3,6 \% \\ 1,5 \% & 2,5 \% & 0,9 \%\end{array}$

4
$22,2 \%$
$66,7 \%$
$1,2 \%$

\section{Outros}

$$
\begin{array}{r}
n \\
\% \text { dentro mecanismo traumático } \\
\% \text { dentro víscera } \\
\% \text { do total }
\end{array}
$$$$
\begin{array}{r}
14 \\
36,8 \% \\
13 \% \\
4,3 \%
\end{array}
$$

$\begin{array}{rr}9 & 14 \\ 28,7 \% & 36,8 \% \\ 8,3 \% & 16,9 \% \\ 2,8 \% & 4,3 \%\end{array}$

0
$0 \%$
$0 \%$
$0 \%$

$\begin{array}{rr}3 & 1 \\ 7,9 \% & 2,6 \% \\ 11,5 \% & 6,3 \% \\ 0,9 \% & 0,3 \%\end{array}$

Total

\begin{tabular}{rrrrrrrr}
$n$ & 94 & 99 & 69 & 6 & 23 & 15 & 347 \\
$\%$ total & $33,2 \%$ & $33,2 \%$ & $25,5 \%$ & $1,8 \%$ & $7,9 \%$ & $4,8 \%$ & $106,4 \%$ \\
\hline
\end{tabular}


Tabela 3 - Trauma perfurante versus Víscera atingida.

\begin{tabular}{|c|c|c|c|c|c|c|c|}
\hline $\begin{array}{l}\text { Lesão } \\
\text { perfurante }\end{array}$ & Baço & Fígado & $\operatorname{Rim}$ & Estômago & Delgado & Cólon & Total \\
\hline \multicolumn{8}{|l|}{ Lesão arma branca } \\
\hline$n$ & 0 & 6 & 2 & 1 & 7 & 2 & 18 \\
\hline \% dentro lesão perfurante & $0 \%$ & $27,3 \%$ & $9,1 \%$ & $4,5 \%$ & $31,8 \%$ & $9,1 \%$ & \\
\hline$\%$ dentro víscera & $0 \%$ & $5,6 \%$ & $2,4 \%$ & $16,7 \%$ & $26,9 \%$ & $12,5 \%$ & \\
\hline$\%$ do total & $0 \%$ & $1,8 \%$ & $0,6 \%$ & $0,3 \%$ & $2,1 \%$ & $0,6 \%$ & $5,4 \%$ \\
\hline \multicolumn{8}{|l|}{ Lesão arma fogo } \\
\hline$n$ & 5 & 8 & 3 & 4 & 4 & 4 & 28 \\
\hline \% dentro lesão perfurante & $27,8 \%$ & $44,4 \%$ & $16,7 \%$ & $22,2 \%$ & $22,2 \%$ & $22,2 \%$ & \\
\hline$\%$ dentro víscera & $4,6 \%$ & $7,4 \%$ & $3,6 \%$ & $66,7 \%$ & $15,4 \%$ & $25,0 \%$ & \\
\hline$\%$ do total & $1,5 \%$ & $2,5 \%$ & $0,9 \%$ & $1,2 \%$ & $1,2 \%$ & $1,2 \%$ & $8,5 \%$ \\
\hline \multicolumn{8}{|l|}{ Total } \\
\hline$n$ & 5 & 14 & 5 & 5 & 11 & 6 & 46 \\
\hline$\%$ do total & $1,5 \%$ & $4,3 \%$ & $1,5 \%$ & $1,5 \%$ & $3,3 \%$ & $1,8 \%$ & $13,9 \%$ \\
\hline
\end{tabular}

Tabela 4 - Outcome versus Tratamento.

\begin{tabular}{|c|c|c|c|c|}
\hline $\begin{array}{c}\text { Outcome } \\
\text { (Mortalidade) }\end{array}$ & $\begin{array}{l}\text { Laparotomia } \\
\text { exploradora* }\end{array}$ & $\begin{array}{l}\text { Tratamento lesão } \\
\text { víscera sólida }\end{array}$ & $\begin{array}{l}\text { Tratamento lesão } \\
\text { víscera oca* }\end{array}$ & Total \\
\hline \multicolumn{5}{|l|}{ Sim } \\
\hline$n$ & 8 & 6 & 1 & 15 \\
\hline$\%$ dentro outcome & $44,4 \%$ & $15,8 \%$ & $2,6 \%$ & \\
\hline$\%$ dentro tratamento & $57,1 \%$ & $10,5 \%$ & $2,4 \%$ & \\
\hline$\%$ do total & $7,1 \%$ & $1,8 \%$ & $0,3 \%$ & $9,2 \%$ \\
\hline \multicolumn{5}{|l|}{ Sim com Reintervenção } \\
\hline$n$ & 0 & 0 & 1 & 1 \\
\hline$\%$ dentro outcome & $0 \%$ & $0 \%$ & $100 \%$ & \\
\hline$\%$ dentro tratamento & $0 \%$ & $0 \%$ & $2,4 \%$ & \\
\hline$\%$ do total & $0 \%$ & $0 \%$ & $0,3 \%$ & $0,3 \%$ \\
\hline \multicolumn{5}{|l|}{ Não } \\
\hline$n$ & 6 & 49 & 34 & 89 \\
\hline$\%$ dentro outcome & $6,9 \%$ & $17,5 \%$ & $12,1 \%$ & \\
\hline$\%$ dentro tratamento & $42,9 \%$ & $86,0 \%$ & $82,9 \%$ & \\
\hline$\%$ do total & $5,3 \%$ & $15 \%$ & $10,4 \%$ & 30,7 \\
\hline \multicolumn{5}{|l|}{ Não com Reintervenção } \\
\hline$n$ & 0 & 2 & 5 & 7 \\
\hline$\%$ dentro outcome & $0 \%$ & $28,6 \%$ & $71,4 \%$ & \\
\hline$\%$ dentro tratamento & $0 \%$ & $3,5 \%$ & $12,2 \%$ & \\
\hline$\%$ do total & $0 \%$ & $0,6 \%$ & $1,5 \%$ & $2,1 \%$ \\
\hline \multicolumn{5}{|l|}{ Total } \\
\hline$n$ & 14 & 57 & 41 & 112 \\
\hline$\%$ do total & $12,4 \%$ & $17,4 \%$ & 12,5 & $42,3 \%$ \\
\hline
\end{tabular}

de imediato à laparotomia exploradora.

A lesão de víscera intra-abdominal, com necessidade de intervenção cirúrgica, foi detetada pelos exames complementares realizados em $47,8 \%$ dos casos.

Em $75 \%$ dos doentes com trauma fechado, tratados cirurgicamente, havia lesões não identificadas. As lesões isoladas do delgado, cólon e fígado foram as menos detetadas pelos meios auxiliares de diagnóstico $(20,5 \%, 13,6 \%$ e $13,6 \%$ dos casos, respetivamente, com $p<0,05)$.

$\mathrm{O}$ tratamento não operatório foi efetuado em $65,3 \%$ dos casos, com o tratamento cirúrgico a ser reservado para os restantes. Verificou-se maior percentagem de intervenção cirúrgica nos doentes com lesão penetrante $(77,5 \%)$ e nos casos em que ocorreu lesão de víscera oca $(87,5 \%)$.

Os doentes que sofreram trauma fechado e lesão de víscera sólida, principalmente hepática, renal e esplénica tiveram, preferencialmente, um tratamento conservador (71,6\%, 74,4\% e 70,6\%, respetivamente). Na abordagem cirúrgica, os procedimentos mais frequentemente realizados foram o packing (no caso do fígado), as rafias e as 
resseções.

Realizou-se laparotomia exploradora sem qualquer intervenção adicional em $12,4 \%$ dos doentes (Tabela 4). Verificou-se mortalidade acrescida nesses casos, na maioria por traumatismo crânio-encefálico.

Houve necessidade de reintervenção cirúrgica em 7,1\% dos casos (maior nos doentes com lesão de víscera oca, reintervencionados em $14,3 \%$ dos casos). Apenas um doente morreu após reintervenção.

Os doentes com lesão isolada de víscera oca tiveram, em média, um internamento mais longo do que os que sofreram lesão de víscera sólida isolada (24,3 dias e 19,9 dias, respetivamente).

Dos pacientes com revised trauma score $<4$, cerca de $86 \%$ sofreram trauma fechado $(p=0,026)-66,7 \%$ dos doentes com RTS < 4 foram submetidos a cirurgia por existência de hemorragia não controlada. Todos os indivíduos com atingimento isolado do estômago, $14,3 \%$ dos que apresentaram atingimento concomitante do fígado e baço e $11,1 \%$ dos que tinham atingimento isolado do cólon apresentaram um revised trauma score $<4$, à admissão.

A mortalidade total no nosso estudo foi de $12 \%$. O trauma fechado foi a causa em $94,9 \%$ dos pacientes que morreram, maioritariamente após acidentes de viação $(20 \%$ por atropelamentos e $17,7 \%$ como ocupante de veículo). A maior parte dos doentes falecidos $(74,1 \%)$ tinha um revised trauma score > 4 à admissão, com $89,1 \%$ dos doentes a apresentar lesão de víscera maciça isolada. A mortalidade foi nula nos casos de lesão penetrante.

\section{DISCUSSÃO}

Os mecanismos que estão na origem de uma lesão traumática são importantes na abordagem diagnóstica e terapêutica de cada caso. Neste estudo, à semelhança de outros, ${ }^{6,7}$ o trauma fechado é mais frequente e resulta, sobretudo, de acidentes de viação e quedas, com maior atingimento de órgãos sólidos (fígado e baço). A lesão penetrante deve ser abordada com particular atenção, pela maior percentagem de lesão de víscera oca subjacente. Como referido por vários autores ${ }^{4,8-11}$ verificámos uma maior dificuldade na deteção destas lesões, ainda que sem mortalidade acrescida, contrariamente a alguns estudos.,8-11.

A FAST e a TC são os exames diagnósticos de eleição $0^{1,5,6,8}$ e, em $41,1 \%$ e em $39,4 \%$ dos casos, respetivamente, não detetaram corretamente todas as lesões, maioritariamente após trauma fechado. As lesões menos detetadas foram as de víscera oca, nomeadamente do delgado e cólon. Estes dados estão de acordo com a literatura, ,,6,8-11 que refere como principais limites destes exames as baixas sensibilidade e especificidade da FAST e o grau variável de sensibilidade da TC na deteção de lesão de víscera oca.

Apesar de alguns autores salientarem algumas vantagens da laparoscopia diagnóstica no contexto de trauma ${ }^{16-18}$ esta não é ainda uma abordagem generalizada.

A maior percentagem de laparotomias exploradoras sem qualquer intervenção adicional verificou-se em doentes vítimas de acidente de viação (como ocupante de veículo). Apesar de 90,9\% dos doentes apresentarem um revised trauma score $>4$ à admissão, verificou-se uma mortalidade acrescida nestes casos. Estes resultados são explicados por uma deterioração rápida do estado dos doentes devido às complicações extra-abdominais associadas (em 97,4\% dos casos por traumatismo crânio-encefálico associado).

O trauma já não é sinónimo de cirurgia, ${ }^{12,15,19}$ verificando-se, um aumento da abordagem terapêutica não-operatória, sobretudo após trauma fechado. Verificámos uma maior percentagem de lesões associadas neste contexto, devendo, por isso, haver uma monitorização da evolução do doente, com particular atenção para sinais indicativos de lesão com necessidade de intervenção cirúrgica. Por outro lado, o tratamento cirúrgico foi a abordagem preferencial após trauma penetrante. A mortalidade nestes casos foi nula, de acordo com o revised trauma score $>4$ à admissão.

A maior percentagem de reintervenção cirúrgica verificada em doentes com lesão de víscera oca resulta da abordagem inicial de controlo de danos, também referida por outros,,$^{6,20} \mathrm{com}$ a realização de ressecções essenciais e criação de estomas temporários do cólon e delgado (em $75 \%$ dos casos). Todos os doentes reintervencionados tinham um revised trauma score $>4$ à admissão, o que está de acordo com a baixa mortalidade verificada (apenas um doente morreu).

\section{CONCLUSÃO}

O trauma abdominal é uma importante causa de morbilidade e mortalidade, pelo que, é de extrema importância o conhecimento do contexto epidemiológico em que se insere. No nosso Centro, de uma forma global, o trauma fechado é o mais prevalente e dele advêm lesões traumáticas relativamente estáveis, com possibilidade de controlo não operatório. Contudo, pela maior probabilidade de lesões ocultas, o doente deve ser alvo de uma monitorização atenta a sinais indicativos de lesão não diagnosticada e com necessidade de terapêutica adicional. Por outro lado, apesar de mais raro, o trauma perfurante é responsável pela grande percentagem dos casos de lesão de víscera oca, geralmente tratada cirurgicamente, com maior necessidade de reintervenção e, por isso, com um tempo de internamento mais longo. A abordagem ao trauma visa, cada vez mais, uma evolução focada no diagnóstico rápido, com métodos menos invasivos e, sempre que possível, um tratamento não operatório, procurando sempre minimizar o risco de lesões não diagnosticadas que possam necessitar de intervenção cirúrgica.

\section{CONFLITO DE INTERESSES}

Nada a declarar.

\section{FONTES DE FINANCIAMENTO}

Nenhum subsídio ou bolsa contribuíram para a realização do trabalho. 


\section{REFERÊNCIAS}

1. Holmes JF, Wisner DH, McGahan JP, Mower WR, Kupperman N. Clinical prediction rules for identifying adults at very low risk for intra-abdominal injuries after blunt trauma. Ann Emerg Med. 2009;54:575-84.

2. Raja A, Zane RD. Initial management of trauma in adults. 2012; 20.9 20.31. [Consultado em 2012 Out 15]. Disponível em: http://www.uptodate.com.

3. Paydar S, Salahi R, Izadifard F, Jaafari Z, Abbasi HR, Eshraghian A, et al. Comparison of conservative management and Laparotomy in the management of stable patients with abdominal stab wound. Am J Emerg Med. 2011;30:1146-51.

4. Faria RG, Almeida AB, Moreira H, Barbosa E, Correia-da-Silva P, Costa-Maia J. Prognostic factors for traumatic bowel injuries: Killing time. World J Surg. 2012;36:807-12.

5. Fleming S, Bird R, Ratnasingham K, Sarker S, Walsh M, Patel B. Accuracy of FAST scan in blunt abdominal trauma in a major London trauma centre. Int J Surg. 2012;10:470-4.

6. Brooks A, Simpson J. Blunt and penetrating abdominal trauma. Abdominal Surg. 2009;27:6.

7. Puskarich MA. Initial evaluation and management of blunt abdominal trauma in adults. 2012. [Consultado em 2012 Out 15] Disponível em: http://www.uptodate.com.

8. McStay C, Ringwelski A, Levy P, Legome E. Hollow viscus injury. J Emerg Med. 2009;37:293-9.

9. Tan K, Liu JZ, Go T, Vijayan A, Chiu M. Computed tomography has an important role in hollow viscus and mesenteric injuries after blunt abdominal trauma. Int J Care Injured. 2010;41:475-8.

10. Campillo-Soto A, Soria-Aledo V, Renedo-Villarroya A, Millán MJ, Flores-Pastor B, Girela-Baena E, et al. Utilidad de la tomografía computarizada para el diagnóstico de lesiones intestinales y mesentéricas en el trauma abdominal cerrado. Cir Esp. 2009;85:341-7.

11. Valcárcel CR, Fuentes FT, Gatica JC, Hermosa AR, Jiménez WV, Díaz
DP, et al. Lesiones gastrointestinales y mesentéricas en el paciente politraumatizado: incidencia, demora diagnóstica y pronóstico. Cir Esp. 2009;86:17-23.

12. Saltzherr TP, van der Vlies $\mathrm{CH}$, van Lienden $\mathrm{KP}$, Beenen LF, Ponsen $\mathrm{KJ}$, van Gulik TM, et al. Improved outcomes in the non-operative management of liver injuries. HPB. 2011;13:350-5.

13. Cooper JG, Smith R, Cooper AJ. The incidence of abdominal injury in patients with thoracic and/or pelvic trauma. Injury Extra. 2005;36:25963.

14. Helling TS, Wilson J, Augustosky K. The utility of focused abdominal ultrasound in blunt abdominal trauma: a reappraisal. Am J Surg. 2007;194:728-33.

15. Gibson DE, Canfield CM, Levy PD. Selective nonoperative management of blunt abdominal trauma. J Emerg Med. 2006;31:215-21.

16. O'Malley E, Boyle E, O'Callaghan A, Coffey JC, Walsh SR. Role of laparoscopy in penetrating abdominal trauma: A systematic review. World $\mathrm{J}$ Surg. 2013;37:113-22.

17. Johnson JJ, Garwe T, Raines AR, Thurman JB, Carter S, Bender JS, et al. Use of laparoscopy in the diagnosis and treatment of blunt and penetrating abdominal injuries: 10-year experience at a level 1 trauma center. Am J Surg. 2013;205:317-20.

18. Schmelzer TM, Mostafa G, Gunter Jr OL, Norton HJ, Sing RF. Evaluation of Selective Treatment of Penetrating Abdominal Trauma. J Surg Educ. 2008;65:340-5.

19. Bruce PJ, Helmer SD, Harrison PB, Sirico T, Haan JM. Nonsurgical management of blunt splenic injury: is it cost effective? Am J Surg. 2011;202:810-16.

20. Smith BP, Adams RC, Doraiswamy VA, Nagaraja V, Seamon MJ, Wisle $\mathrm{J}$, et al. Review of abdominal damage control and open abdomens: Focus on gastrointestinal complications. J Gastrointestin Liver Dis. 2010;19:425-35. 


\section{Lesão Visceral em Trauma Abdominal: Um Estudo Retrospetivo \\ Acta Med Port 2013:26:725-730}

Publicado pela Acta Médica Portuguesa, a Revista Científica da Ordem dos Médicos

Av. Almirante Gago Coutinho, 151

1749-084 Lisboa, Portugal.

Tel: +351218428215

E-mail: submissao@actamedicaportuguesa.com

www.actamedicaportuguesa.com

ISSN:0870-399X | e-ISSN: 1646-0758

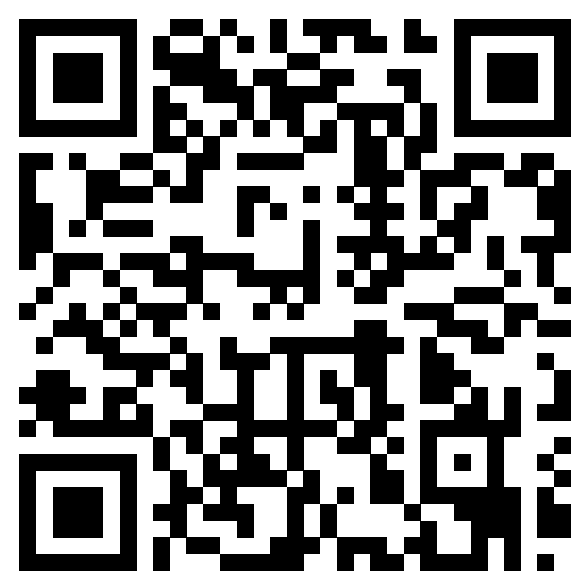

